\title{
A Case of Postoperative SMART Syndrome in a Medulloblastoma Survivor
}

\author{
Cristian Cirjan ${ }^{1} \quad$ Ali Jamal $^{2} \quad$ Philippe Mercier $^{3} \quad$ Rachel Berkovich $^{4}$ Pournima Navalkele ${ }^{5}$
}

${ }^{1}$ Cardinal Glennon Children's Hospital, Saint Louis University School of Medicine, Saint Louis, Missouri, United States

2 Department of Neurology, Cardinal Glennon Children's Hospital, Saint Louis University, Saint Louis, Missouri, United States

${ }^{3}$ Department of Neurosurgery, Cardinal Glennon Children's Hospital, Saint Louis University, Saint Louis, Missouri, United States

${ }^{4}$ Department of Radiology, Cardinal Glennon Children's Hospital,

Saint Louis University, Saint Louis, Missouri, United States

${ }^{5}$ Division of Hematology Oncology, Department of Pediatrics, Cardinal Glennon Children's Hospital, Saint Louis University, Saint Louis, Missouri, United States

Address for correspondence Pournima Navalkele, MD, 1465 South Grand Boulevard, Saint Louis, MO 63104, United States (e-mail: Pournima.navalkele@gmail.com).

J Child Sci 2021;11:e327-e330.

\begin{abstract}
Keywords

- radiation

- migraine

- stroke

- SMART syndrome

Stroke-like migraine attacks after radiation therapy (SMART) syndrome is a rare neurological phenomenon characterized by reversible symptoms of headache, seizure, hemiparesis, and visual changes associated with a remote history of cranial irradiation. We describe a case of a medulloblastoma survivor, presenting with postoperative neurological deficits, along with neuroimaging findings and subsequent resolution of symptoms, highly suggestive of SMART syndrome. We have also reviewed the common epidemiological and diagnostic factors associated with this rare disease, as well as discussed pathophysiological mechanisms.
\end{abstract}

\section{Introduction}

Stroke-like migraine attacks after radiation therapy (SMART) syndrome is a rare neurological complication that may appear months to years after radiotherapy with characteristic clinical signs such as hemiparesis, seizures, headache, nausea, vomiting, and visual disturbances. Magnetic resonance imaging (MRI) scan typically demonstrates prominent unilateral gyriform enhancement on postcontrast imaging, a hyperintense signal on $\mathrm{T} 2$ and fluid-attenuated inversion recovery (FLAIR)-weighted sequences. ${ }^{1,2}$ We describe a case of a 26-year-old medulloblastoma survivor, presenting with postoperative neurological deficits, along with neuroimaging findings and subsequent resolution of symptoms, highly suggestive of SMART syndrome. This case report is only the second to describe SMART syndrome as a possible postoperative phenomenon, an important association that may dramatically impact the clinical management.

\section{Case Report}

A 26-year-old male patient presented to the long-term cancer survivors' clinic for annual follow-up for medulloblastoma diagnosed at 4 years of age. Initial treatment was gross total resection of the tumor from the cerebellum followed by adjuvant therapy based on Children's Oncology Group A9961 protocol consisting of craniospinal irradiation $36 \mathrm{~Gy}$ with posterior fossa boost to $54 \mathrm{~Gy}$, followed by chemotherapy consisting of vincristine, lomustine, and cisplatin. After completion of therapy, he developed chronic migraines, bilateral sensorineural hearing loss secondary to chemotherapy, hypothyroidism, and cognitive delay, but was received

August 27, 2021

accepted after revision

November 2, 2021
DOI https://doi.org/

10.1055/s-0041-1740466.

ISSN 2474-5871. (c) 2021. The Author(s).

This is an open access article published by Thieme under the terms of the Creative Commons Attribution License, permitting unrestricted use, distribution, and reproduction so long as the original work is properly cited. (https://creativecommons.org/licenses/by/4.0/)

Georg Thieme Verlag KG, Rüdigerstraße 14, 70469 Stuttgart, Germany 
never diagnosed with hypertension or seizures. On this visit, he reported no new complaints, and the clinical examination demonstrated known cognitive deficits, hearing loss, and ataxia. MRI of the brain, done as a part of the annual evaluation, was concerning for numerous new enhancing lesions involving the left occipital lobe (largest of all lesions, restricting diffusion), thalami, and cerebellum. MRI of the brain angiogram did not show any aneurysm or stenosis. The patient was urgently referred to neurosurgery for biopsy of the occipital lesion, given the imaging concern for secondary malignancy. The neurosurgeon performed an open biopsy due to the relatively small area of enhancement and superficial nature of the lesion in the noneloquent cortex and due to concerns that a needle biopsy may not help clinch a diagnosis. Immediate postoperative MRI of the brain showed expected changes of diffusion restriction at the biopsy site and a small subdural collection. Five days after discharge, the patient developed confusion, headache, visual deficit, leg weakness, and an episode of generalized shaking that began 2 days prior. His headaches consisted of brief moments of severe pain lasting a few seconds, typically occurring in the morning and afternoon, which were different from his prior chronic migraines. His examination revealed new-onset disorientation, right hemianopsia, and worsening ataxia. MRI of the brain demonstrated new extensive gyriform contrast enhancement and diffusion restriction along the surfaces of the left occipital lobe (-Fig. 1). Magnetic resonance angiogram, venogram, and spectroscopy did not show any abnormalities. Electroencephalogram confirmed focal electrographic seizures, necessitating the initiation of antiepileptic drugs. The differential diagnosis included leptomeningeal tumor spread, meningoencephalitis, postoperative ischemic insult, and SMART syndrome, all of which can have cortical and leptomeningeal enhancement on MRI. ${ }^{3}$ Pathology of the previously obtained occipital lesion biopsy showed multifocal vasculopathy and microglial activation, without evidence of neoplasia, thus ruling out leptomeningeal metastasis. Meningoencephalitis was ruled out as cerebrospinal fluid (CSF) studies demonstrated negative cultures and absence of inflammatory cells. Ischemic insult was unlikely, given the delayed onset of symptoms relative to the postoperative period as well as the absence of expected ischemia on imaging (not confined to an arterial vascular distribution and not associated with venous injury or thrombosis to suggest venous infarct). Additionally, several other differential diagnoses were ruled out ${ }^{3}$ such as posterior reversible encephalopathy syndrome, given the lack of bilateral MRI findings. Mitochondrial encephalopathy, lactic acidosis, and stroke syndrome was ruled out due to the absence of lactic acidosis and absence of any family history. A lack of CSF lymphocytic pleocytosis made transient headache and neurological deficits with CSF lymphocytosis less likely, and reversible cerebral vasoconstriction syndrome was ruled out due to the absence of vascular narrowing on magnetic resonance angiography. The patient's neurological status improved gradually with a near-complete resolution of new deficits within a month, without the use of steroids. Concurrently, the MRI of the brain showed dramatic resolu-
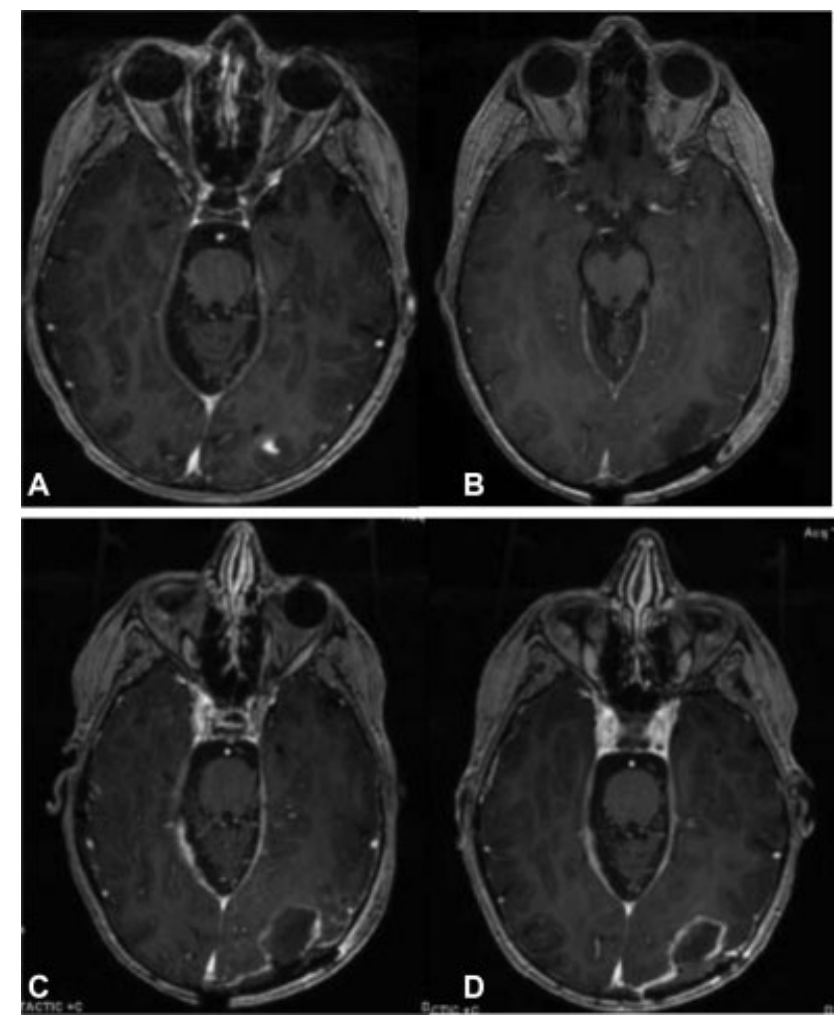

Fig. 1 Magnetic resonance imaging of the brain with axial postcontrast images showing (A) contrast-enhancing left occipital lesion, (B) immediate postoperative image showing gross total resection of the occipital lesion, (C) subacute-onset new leptomeningeal enhancement in left occipital lobe, and (D) resolution of leptomeningeal enhancement in the left occipital lobe.

tion of the extensive leptomeningeal enhancement and diffusion restriction (-Fig. 2) along with stability in the previously known enhancing lesions in the thalami and cerebellum.

\section{Discussion}

\section{Diagnosis and Epidemiology}

Shuper et al were the first to document the complicated "migraine-like episodes" in four children following cranial irradiation and chemotherapy. ${ }^{4}$ After 1.2 to 2.8 years following diagnosis, these patients presented with new-onset, severe, intermittent unilateral headaches associated with nausea, hemiparesis/hemisensory loss, episodic visual loss, and aphasia, usually lasting only 2 to 24 hours. With two additional patients presenting with similar symptoms, Black et al proposed the following criteria for the diagnosis of SMART syndrome: (1) history of brain irradiation for cancer, (2) reversible symptoms such as headache, seizure, hemiparesis, and visual changes, (3) characteristic reversible MRI findings of gyriform contrast enhancement, with abnormal T2 and FLAIR signal hyperintensities in the previous irradiation port sites, and (4) lack of evidence of residual or recurrent tumor and a lack of other attributable causes to other diseases. ${ }^{5}$ Although the syndrome is considered to be reversible within 2 to 5 weeks, a follow-up case series with 11 patients revealed permanent neurological deficits and 

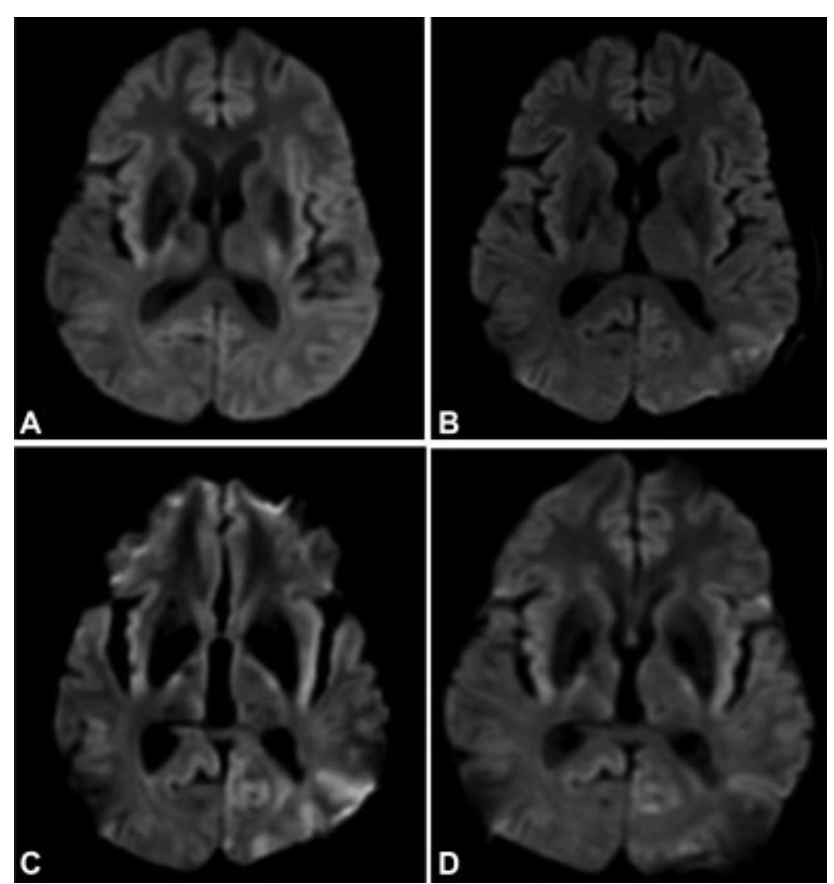

Fig. 2 Magnetic resonance imaging of the brain with axial diffusionweighted images showing $(\mathrm{A})$ preoperative imaging showing no diffusion restriction in the left occipital lobe (above the level of the enhancing lesion), (B) immediate postoperative image showing no diffusion restriction in the left occipital lobe, $(C)$ subacute-onset new restricted diffusion in the left occipital lobe, and (D) resolution of restricted diffusion in the left occipital lobe.

imaging findings. ${ }^{6}$ Specifically, $55 \%$ of these patients achieved symptom resolution after 2 months, while the remaining $45 \%$ maintained some permanent neurological deficits, manifesting as dysphagia, cognitive impairment, and hemiparesis. Our patient met all the diagnostic criteria for SMART syndrome, and his symptoms did reverse after a month. In the largest review to date, Singh et al analyzed 25 patients with 31 episodes of SMART syndrome, generating additional findings supportive of the diagnosis, ${ }^{7}$ notably, CSF glucose, protein, and cell count are typically within normal limits in all patients, as was evident in our patient. As described in the case series by Shuper et al, the four patients who underwent biopsy of the lesions found on MRI were found to have nonspecific gliosis. Similarly, our patient had nonspecific gliosis in the biopsy of the occipital lesion.

Additionally, the most common deficits associated with SMART syndrome were migraine headaches (70.9\%), focal deficits (93.5\%), generalized tonic-clonic (GTC) seizures (75\%), encephalopathy (70.9\%), and visual disturbances (70.9\%). Our patient's confusion, GTC seizures, and visual disturbance similarly aligned with these findings. Epidemiologically, most SMART episodes take place at an average of 21.6 years following initial radiation. Our patient developed these symptoms 22 years after radiation treatment. Singh et al so noted that 5 out of 25 patients also developed infarcts, mostly in the temporal and occipital regions associated with gyriform enhancement. Diffusion restriction along the area of gyriform enhancement was also seen on our patient's MRI, making infarction a possible component of SMART syndrome.

This patient is notably the second documented case of SMART syndrome likely induced after neurosurgery. Maloney et al reported the first case involving a 56-year-old woman who underwent temporal lobectomy for recurrent adenocarcinoma metastasis. ${ }^{8}$ Unlike our case, their patient reported painful migraine-like symptoms immediately postoperatively with left spastic hemiparesis and hemineglect, lasting 2 days.

\section{Pathophysiology}

Although the pathophysiology of SMART syndrome remains poorly understood, some authors have speculated that deranged autoregulation caused by endothelial damage may be a causative factor. ${ }^{6,7}$ In their review of radiotherapy-induced vasculopathy, Murphy et al specifically identified cerebrovascular accidents, moyamoya syndrome, vascular malformation, and hemorrhage as some of the principal vascular pathologies that take place years following cranial irradiation. ${ }^{9,10}$ Given the high incidence of infarcts (not in an arterial distribution) demonstrated by Singh et al as well as the contrast enhancement found in most cases, it is thought that radiotherapy-induced vasculopathy plays a role in the pathogenesis of SMART syndrome.

Another proposed pathophysiological mechanism is disruption of the trigeminovascular system, which has previously been implicated in the pathophysiology of headaches and vasospasm, given the multiple potent vasodilator peptides found within its neurons, including calcitonin generelated peptide, substance $\mathrm{P}$, and neurokinin $\mathrm{A} .{ }^{8}$ In the only other case of a surgically induced SMART syndrome, the authors postulated that the resection of the dura overlying the Gasserian ganglion could have manipulated an already dysfunctional trigeminovascular system, setting off SMART syndrome.

\section{Conclusion}

Although SMART syndrome has been previously described in the literature, this is the second documented case describing its occurrence after an open biopsy, leading to the possibility that intracranial surgery may precipitate SMART syndrome in susceptible patients with prior history of cranial irradiation. Given that the management paradigm for SMART syndrome contrasts sharply with its mimics, such as postoperative ischemic insult, meningoencephalitis, or leptomeningeal metastasis, making the correct diagnosis has vital clinical implications.

Note

Written informed consent for publication of the clinical details was obtained from the legal guardian. A copy of the consent form is available for review by the editor of this journal.

Funding

None. 
e330 Radiation, Migraine, Stroke, and SMART Syndrome Cirjan et al.

\section{Conflict of Interest \\ None declared.}

\section{References}

1 Makary MS, Awan U, Puduvalli VK, Slone HW. Stroke-like migraine attacks after radiation therapy syndrome: clinical and imaging characteristics. J Clin Imaging Sci 2019;9:5

2 Khanipour Roshan S, Salmela MB, McKinney AM. Susceptibility-weighted imaging in stroke-like migraine attacks after radiation therapy syndrome. Neuroradiology 2015;57(11): 1103-1109

3 Dominguez M, Malani R. Stroke-like migraine attacks after radiation therapy (SMART) syndrome: a comprehensive review. Curr Pain Headache Rep 2021;25(05):33

4 Shuper A, Packer RJ, Vezina LG, Nicholson HS, Lafond D. 'Complicated migraine-like episodes' in children following cranial irradiation and chemotherapy. Neurology 1995;45 (10):1837-1840
5 Black DF, Bartleson JD, Bell ML, Lachance DH. SMART: stroke-like migraine attacks after radiation therapy. Cephalalgia 2006;26 (09):1137-1142

6 Black DF, Morris JM, Lindell EP, et al. Stroke-like migraine attacks after radiation therapy (SMART) syndrome is not always completely reversible: a case series. AJNR Am J Neuroradiol 2013;34(12):2298-2303

7 Singh TD, Hajeb M, Rabinstein AA, et al. SMART syndrome: retrospective review of a rare delayed complication of radiation. Eur J Neurol 2021;28(04):1316-1323

8 Maloney PR, Rabinstein AA, Daniels DJ, Link MJ. Surgically induced SMART syndrome: case report and review of the literature. World Neurosurg 2014;82(1-2):240.e7-240.e12

9 Murphy ES, Xie H, Merchant TE, Yu JS, Chao ST, Suh JH. Review of cranial radiotherapy-induced vasculopathy. J Neurooncol 2015; 122(03):421-429

10 Armstrong AE, Gillan E, DiMario FJ Jr. SMART syndrome (strokelike migraine attacks after radiation therapy) in adult and pediatric patients. J Child Neurol 2014;29(03):336-341 